\title{
IDSC I.AIMFiR
}

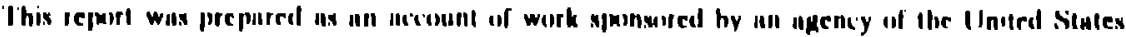

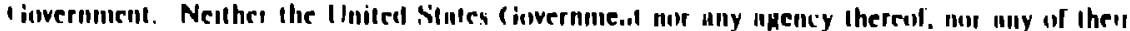

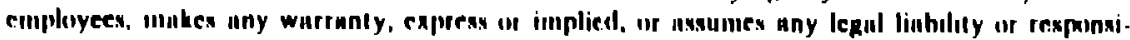

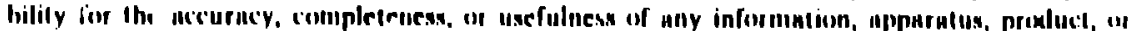

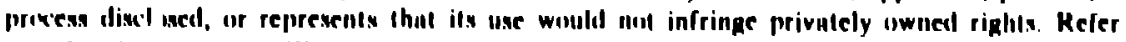

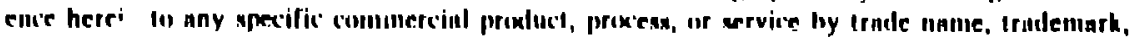

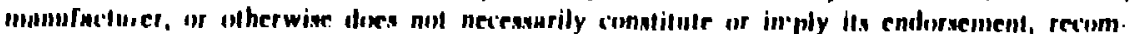

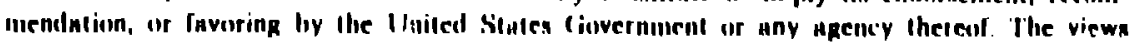
und opinions of nuthors expresued herein do nut necessarily state of reflect thowe of the IInital Simten therernment or moy naene?; theress.
\end{abstract}




\title{
COLLECTIVE ION ACCELERATION BY MEANS OF VIRTUAL CATHODES
}

\author{
William Peter, Rickey J. Fachl, Charles Snell, and Michael E. Jones \\ Los Alames National Laboratory \\ University of Ca!ifornia \\ Los Alamos, New Mexico 87545
}

\section{Introduction}

Experiments on collective ion acceleration by means of the formation of a virtual cathode have been carried out for a number of years in the Soviet Union and in the United Staies. Recently, there has been renewed interest in the subject as a possible means of acceleratiog ions to very high energies. By understanding the physics underlying the acceleration process it may be possible $t$ s determine the feasability of virtual cathode staging for very high energy ion production.

For this reason, a theoretical and computational effort is underway at Los Alamos in order to clarify the tasic issues of collective ion arceleration by means of virtual cathodes. To support the theoretical eflort, simulations were done with the fully electromagnetic and relativistic particlo-in-coll code ISIS (in a one-dimensional mode) and the electrostatir one-dimensional code BICON!: In the simulations, an electron beam of density $6 \times 10^{11} \mathrm{~cm}^{-3}$ is injected into a che-dimensional box of length $L$. To supply the necessary ions for collective acceleration, a pla:ma source containing both ions and electrons was initialized near the emitting boundary (Fig. 1).

Of prime interest in this study was to understand the dyonics of virtual cathode formation and the dynamics of the acceleration process for the ions. In particular, the guestiun of whether the ions are accelerated by a moving potential well or hydrodynamic pressure due to nmbipolar expansion 2 is of primary interest. 


\section{Virtual Cathode Formation}

Consider a relativistic electron beam injected into a box of length $L$ such that $\varphi(x=0)=0$ and $\varphi(x=L)=0$. A time-dependent analysis of the problem up to the first electron turning point was presented by Poukey and Rostoker for the one-dimensional problem, but with the boundary conditions $\varphi(x=0)=0$ and $E(x=\infty)=0$. Even so, their results should describe the problem at hand if $x_{m} / L \rightarrow 0$, where $x_{m}$ is the position of the virtual catbode.

To study the formation and dynamics of the formation of a virtual cathode, simulations were done in order to verify a simple one-dimensional steady-state model, and to provide a benchmark for understanding the general time-dependent problem. In the simulations, electrons are injected into a drifl-tube consisting of two grounded end plates. Plots of the electric potential, electric field, and virtual cathode position were obtained for different values of the injection velocity.

According to the timedependent theory, a virtual cathode should form at a distance on the order of an electron beam skin depth $c / \omega_{6}$ from the emitter. The earliest electrons should rropagate across the box at the injection velocity "0. This effect could be easily seen in the simulations. After the beam front leaves the box, the gross features of the phase space are constant, though the simulations definiteiy show vitual cathode oscillations. The oscil-

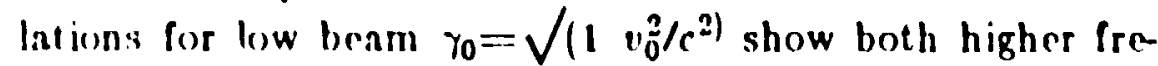
quenc." and amplitude than the corresponding simulations for large $\gamma_{0}$.

'1!e position of the virtual cathode was also measured as a function of the beam $\gamma_{0}$ for different values sf the beam injection "elocity. To minimize the effects of the oscillations in the virtual cathode position, these runs were dene for large values of $\gamma_{0}$. As Fig. 2 shows, the agresment between the simulations and the Poukey-Rostoker theoretical sealing $\left(s_{m}\right.$ proportional to $\left.\gamma_{0}^{l^{m}}\right)$ is quite good.

For late times in the simulations, a "stendy state" was reached. By this wo mean all physieal qunntities averaged over the virtual cathode oscillntions were essentinlly unchanged for all time. A simple, atendy-state model war lhen formelated which sasumes a priori that a virtual calhede forms at the point $x-x_{m}$. Assuming the beam makey up three species (the injectid partictes, the reflected 
particles, and the transmitted particles), Poisson's equation, the equation of continuity, and the conservation of energy equation $\left(\gamma-\gamma_{0}\right) m c^{2}=e \varphi$ for each species can be used. The solutions cannot be integrated exactly in the general case, but in the non-relativistic regime they can be obtained exactly. The result for the potential $\bar{\varphi}\left(\equiv \operatorname{e\varphi } /(\gamma-1) m e^{2)}\right.$ can be written:

$$
\bar{\varphi}(\bar{x})=[1-3 \bar{x} \sqrt{ }(1-T / 2)]^{43}-1
$$

for $x<x_{m}$, and

$$
\bar{\varphi}(\bar{x})=[-\bar{L}-\bar{x}) \sqrt{ }(g T / 2)]^{\sqrt{3}}-1
$$

for $x>x_{m}$. In Eqs. (2) the dimensionless lengths are overstruck by bars and are normalized to the beam skin depth $\delta=v / w_{b}$. The quantity $T$ (which is a function of $\bar{x}_{m}$ only) is the transmission coefficient of the beam; that is, the ratio of the transmitted current to the injected current. In Fig. 3, Eq. ( 2$)$ is plotted in comparison with a typical potential plet. from the one-dimensional simulations. The agreement is surpisingiy good.

The steady-state solutions above impose a requirement on the virtual cathode position as a function of the initial beam density $n_{0}$, the initial beam velocity $v_{0}$, and the length L. of the gap spacing. In terms of dimensionless quantities, this equation can be written:

$$
\bar{L}=\bar{x}_{m}+\left(0 \cdot 1 / \bar{x}_{m}^{2}\right)^{-1 / 2}
$$

In Fig. I we plot the theoretical curve for Fq.(3) with the corresponding simulation data points. The simulations were made with values of $I$, ranging from $0.1 \mathrm{~cm}$. Identical values for the initinl beam density $n_{0}$ and velocity $v_{0}$ were used in these runs. Buenuse of the variation in virtual cathode position due to the virtual cathode oscillations in this nonrelativistic case ( $\left.\gamma_{0}=1.01\right)$, the data points correspond to a simple nverage of the maximum and minimum amplitudes.

\section{Collective Acceleration}


When a plasma is introduced in to the box, the beam space charge can be neutralized by this plasma. The beam then propagates a distance equal to $c / \omega_{b}$ beyond the plasma boundary, until a virtual cathode forms. In the "moving virtual catbode theory", the resulting electron space charge in the thin sheath beyond the plasma pulls the ions ahead in a bootstrap process. The motion of the potential well with respect to time c.un be derived in a simple fastion from Eq.(2), and will be discuss ad in a later study.

In the onedimensional runs with an ion-to-electron mass ratio of 1836, no virtual cathode motion was seen. Typically, these runs bad a rich source of ions in the form of a localized plasma of density 50 times the injected electron beam density. When the electron-to-ion mass raţio was reduced to 20 , as in the work of Mako and Tajima, there was virtual cathode motion observed. Whether or not the virtual catbode movement is simply a result of the artificially low mass ratio or a real physical clfect has not yet been determined.

The one-dimensional simulations with equipotential boundary conditions $(\varphi=0)$ show that the measured maximum ion cnergy is a little over three times the initial beam energy (Fig. 4). This agrees with the results of Ref. 3. In the simulations using the Poukey and Rostoker boundary couditions, the measured maximum ion energy increases up to twenty times the initial beam energy. In the two dimensional simulations the maximum ion energy scaled as $\left(I / I_{S C L}\right)^{1 / 2}$, where $I_{S C l}$ is the space-charge limit current in the drift tube.

In conclusion, we have presented a simple calculation for the electric potentina and electric field in a one dimensional virtual cathode formed by the injection of a relativistic electron bean into a drift tube region. The theoretical predictions serm to agree in a time-averaged sense with a simple steady statc model. The maximum energy given to the ions is a little over three times the initial electron beam energy, in ngerement with the results of previous nuthors. Further work is needed to extend the simple analytic model to relativiutic, and perhaps timedependent, regimes. Finally, it is planned to use the results of this stu!y to design future ancelerators with signiferantly higher values of ion rorrgy. 


\section{Acknowledgments}

We would like to thank Abraham Kadish and Don Lemons of LANL, and Rick Mako of the Naval Research Laboratory for important and helpful discussions.

\section{References.}

1. J.W. Poukey and N. Rostoker, Plasma Physics 13, 887 (1871).

2. R.J. Faehl, W.K. Peter, this conference.

3. F. Mako and T. Tajima, Phys. Fluids 17, 1815 (1884). 
Figure captions.

Fig. 1. Schematic drawing of a relativistic electron beam injected in to a drift region containing a localized source of plasma. The collector plate, which is at a distance of $L \mathrm{~cm}$ away from the emitter, is not shown.

Fig. 2. Virtual cathode position $x_{m}$ as a function of initial beam energy $\gamma_{0}$. The solid curve shows $\gamma_{0}^{1 / 2}$ scaling.

Fig. 3. Potential plots.

Fig. 4. Variation of virtual cathode postition $x_{m} / \delta$ as an implicit function of the gap spacing $L / \delta$ from Eq. (3). The data points are from one-dimensional simulations.

Fig. 5. Ratio of maximum ion energy to initial beam energy as a function of $\nu / \gamma$. 
PHI VS. X

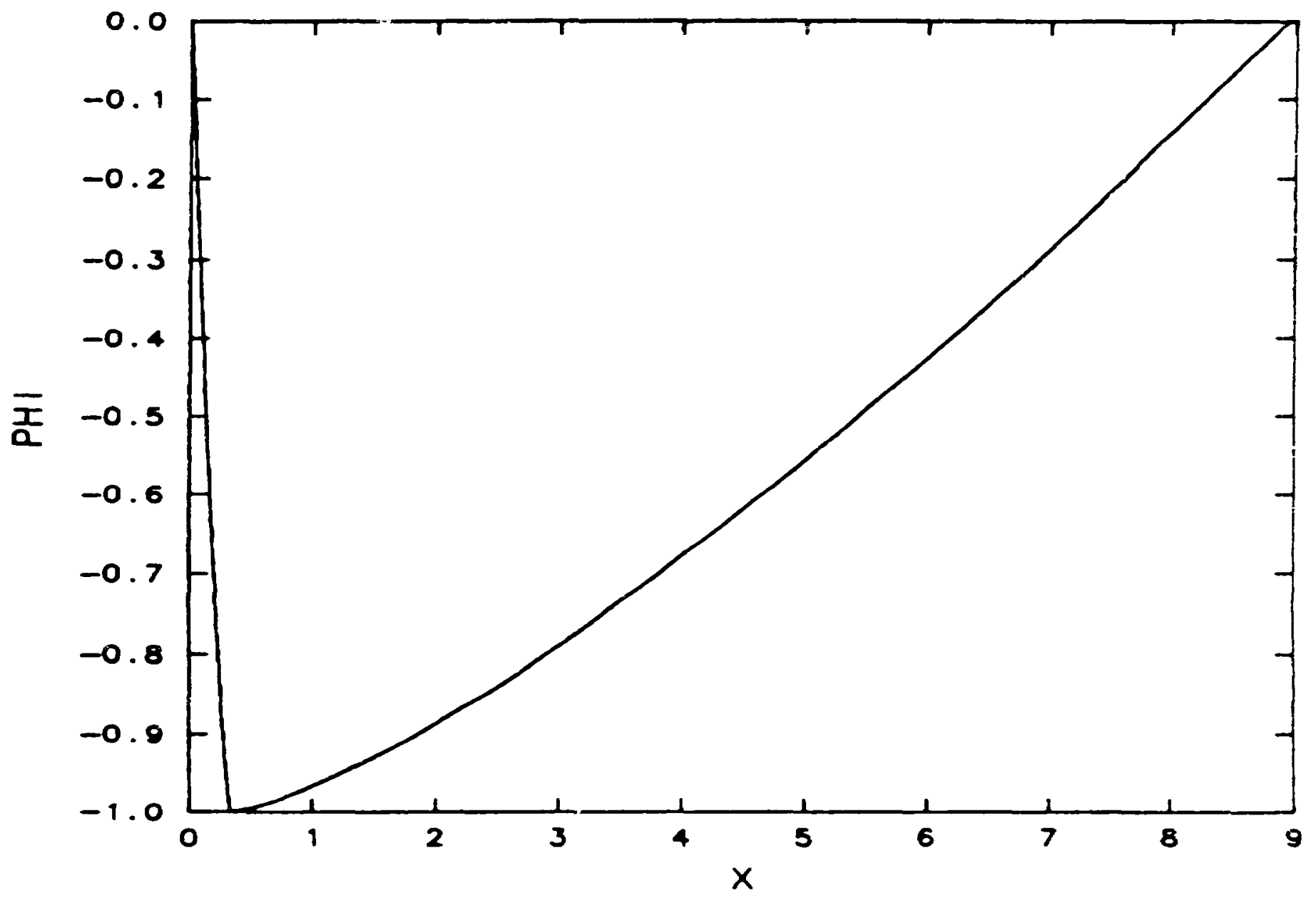


PHI VS. $X$

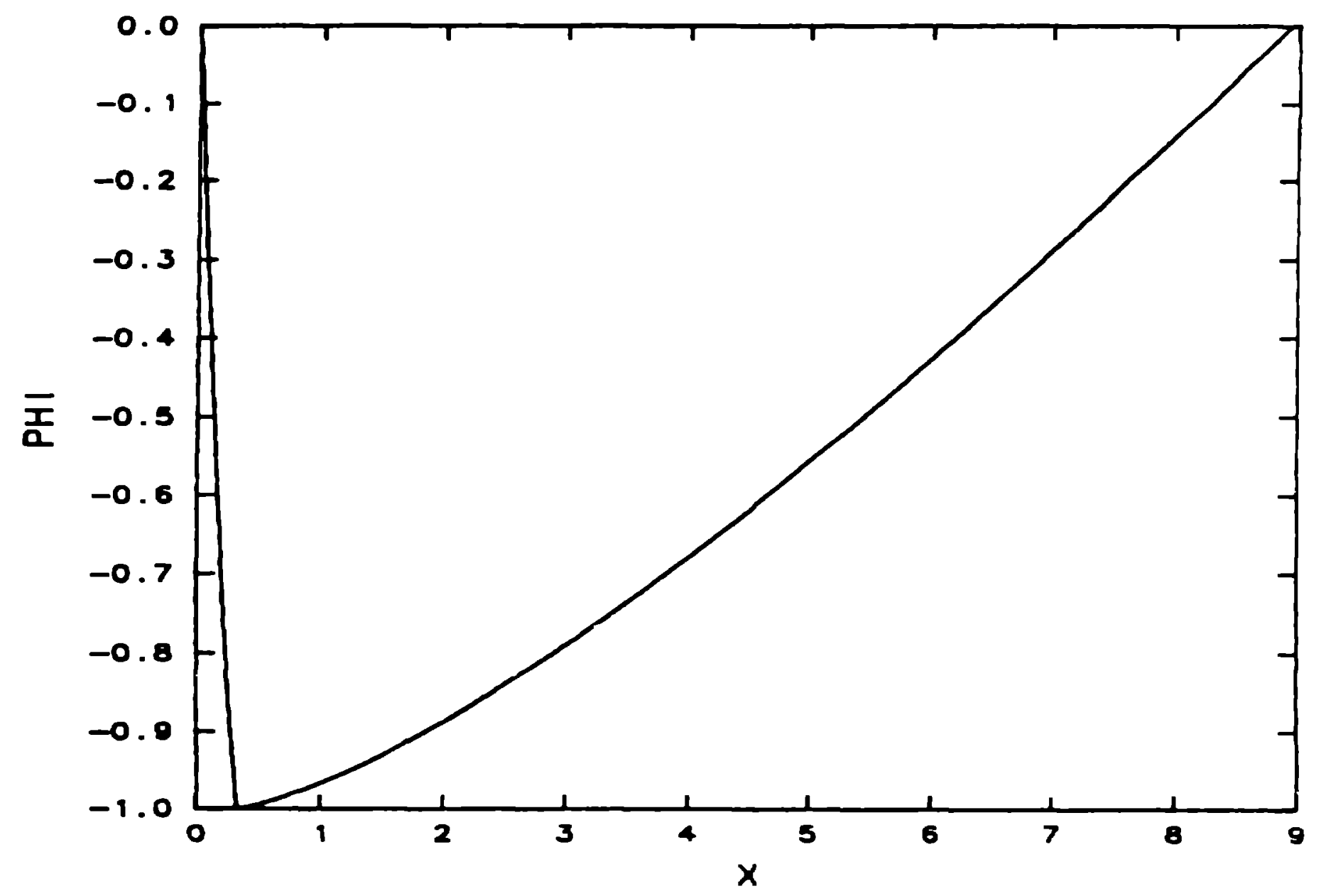




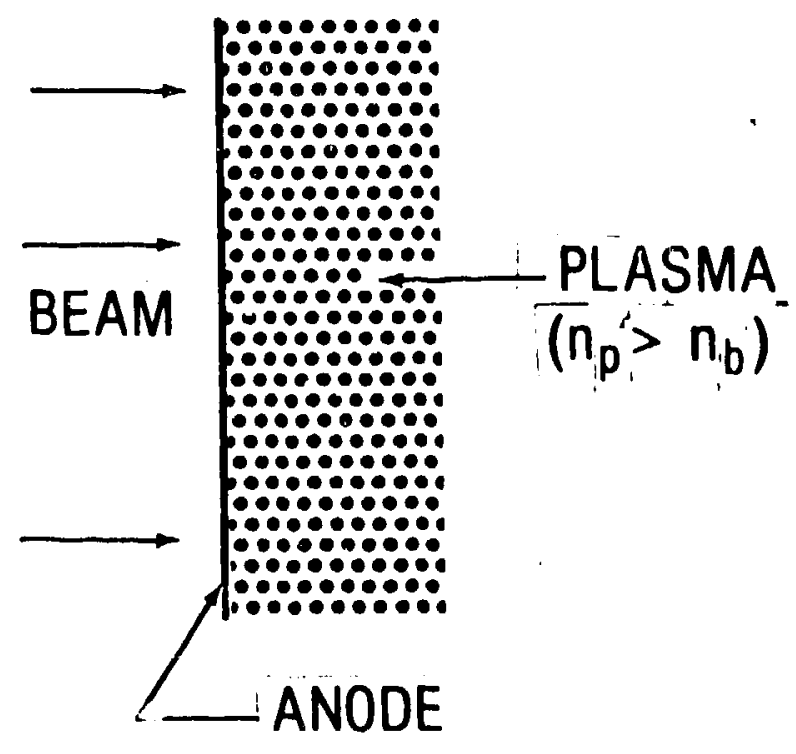




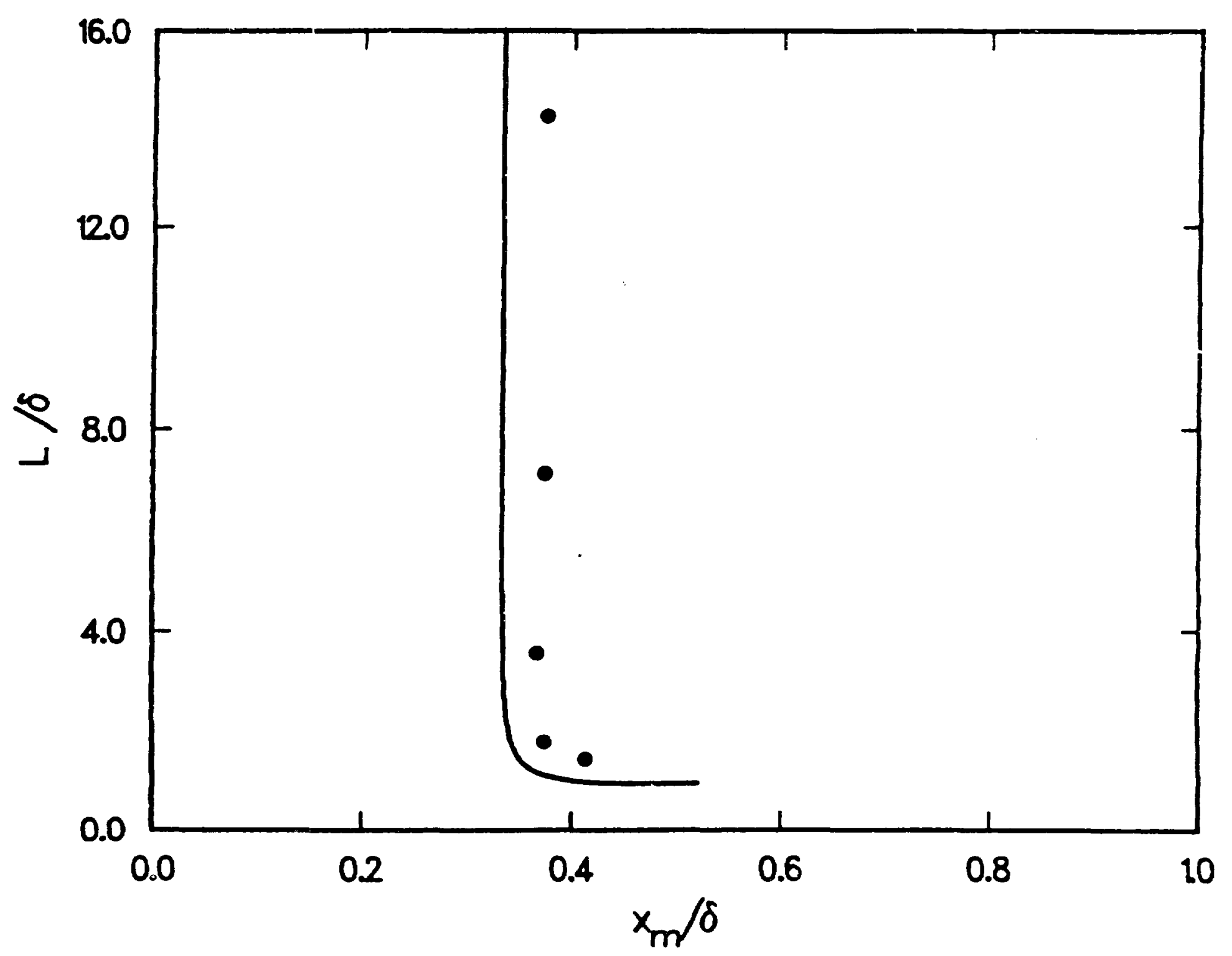




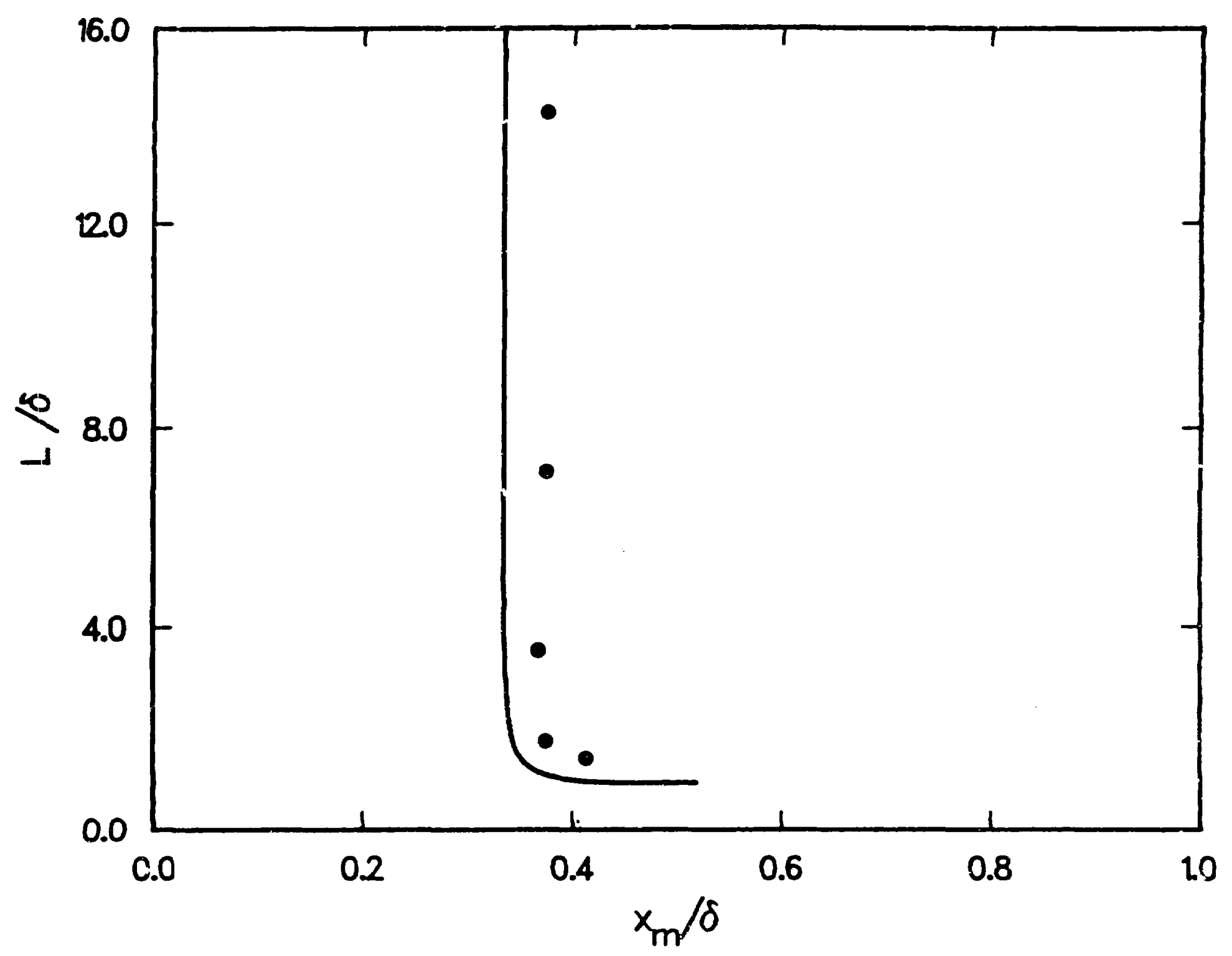




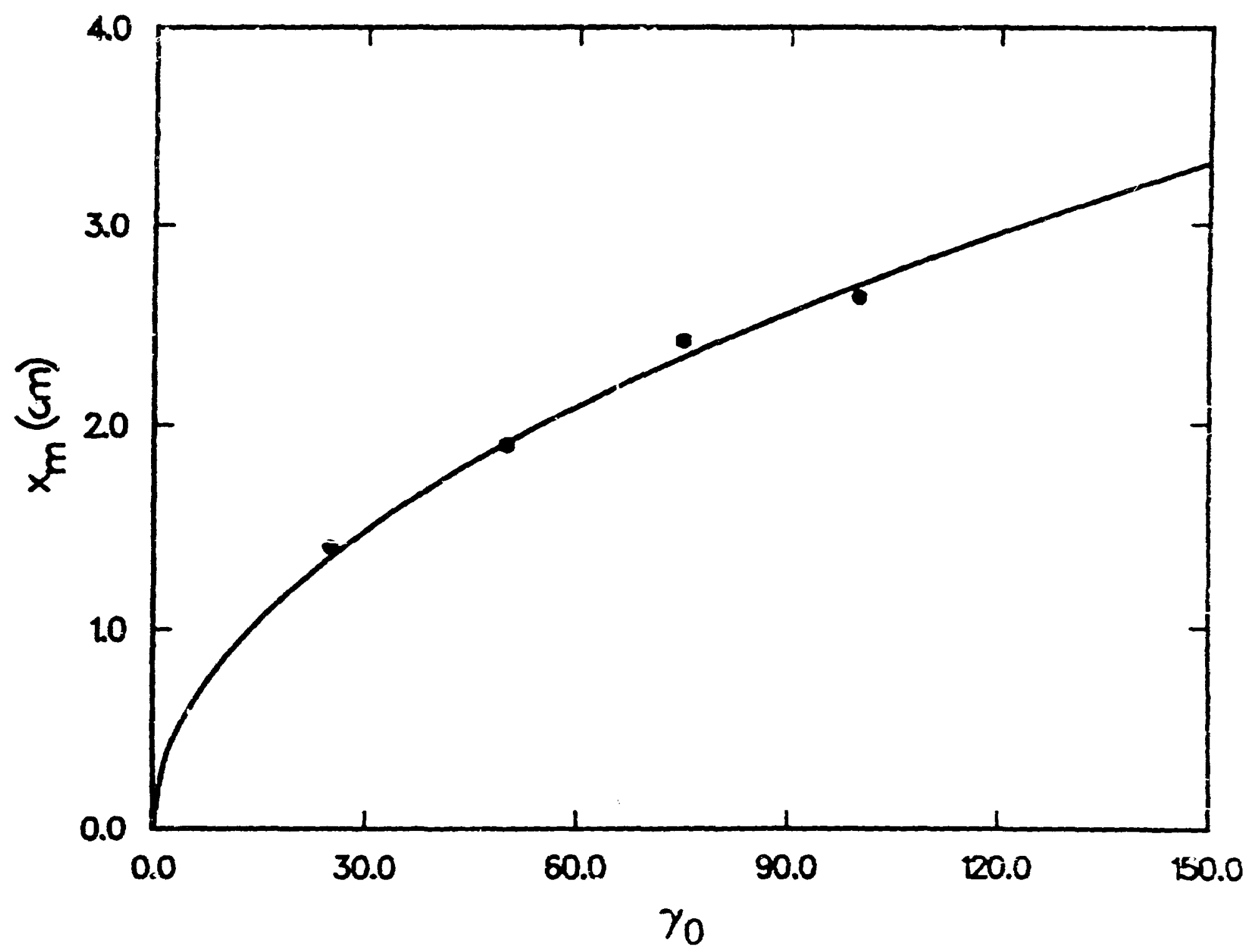




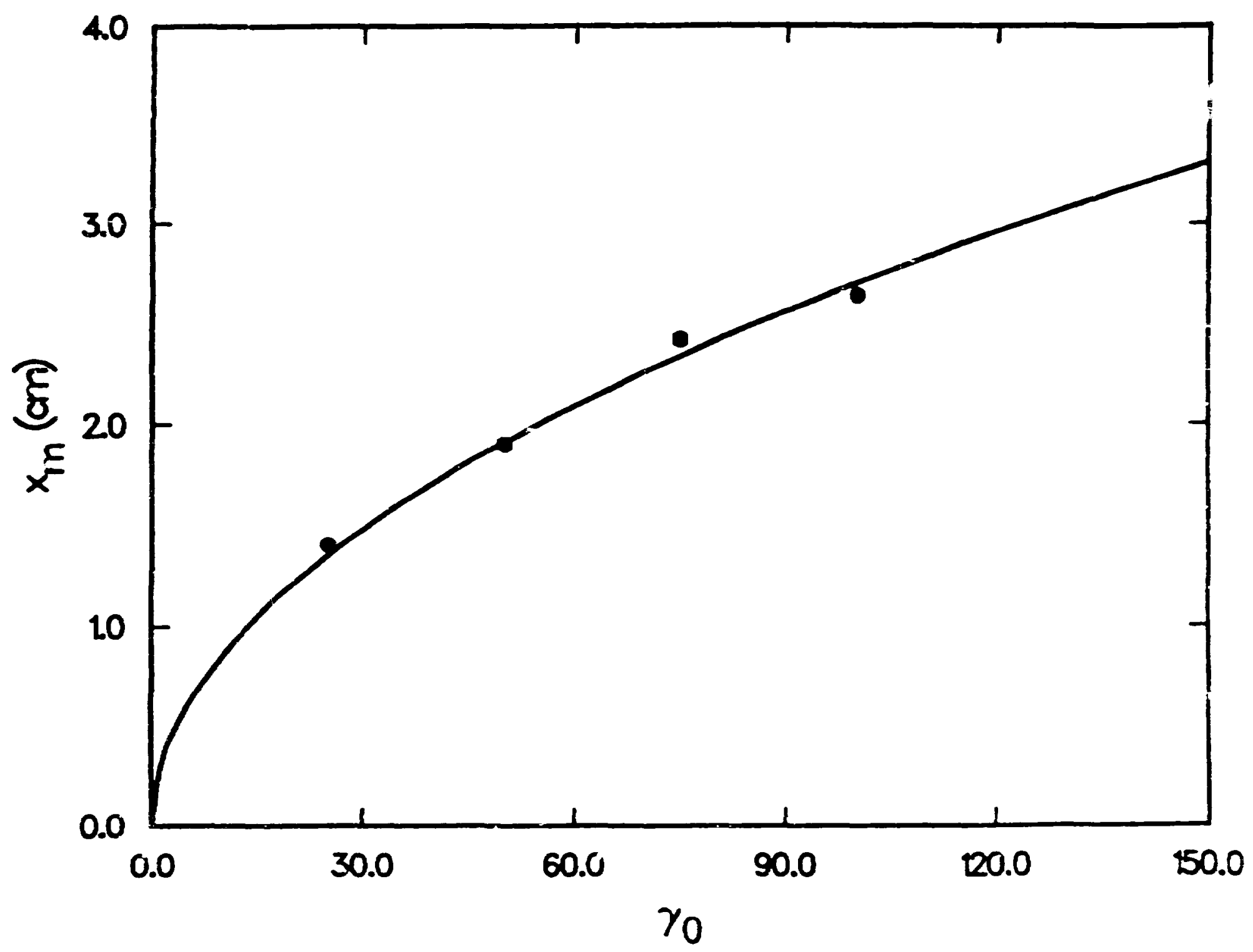

